University of Louisville

ThinkIR: The University of Louisville's Institutional Repository

Faculty Scholarship

$6-2014$

\title{
The baryonic Tully-Fisher relationship for S4G galaxies and the "condensed" baryon fraction of galaxies.
}

\author{
Dennis Zaritsky \\ University of Arizona \\ Helene M. Courtois \\ Universite Lyon \\ Juan Carlos Munoz-Mateos \\ National Radio Astronomy Observatory \\ Jenny Sorce \\ Universite Lyon \\ S. Erroz-Ferrer \\ Instituto de Astrofísica de Canarias \\ See next page for additional authors
}

Follow this and additional works at: https://ir.library.louisville.edu/faculty

Part of the Astrophysics and Astronomy Commons

\section{Original Publication Information}

Zaritsky, Dennis, et al. "The Baryonic Tully-Fisher Relationship for S4G Galaxies and the "Condensed" Baryon Fraction of Galaxies." 2014. The Astronomical Journal 147(6): 11 pp.

This Article is brought to you for free and open access by ThinkIR: The University of Louisville's Institutional Repository. It has been accepted for inclusion in Faculty Scholarship by an authorized administrator of ThinkIR: The University of Louisville's Institutional Repository. For more information, please contact thinkir@louisville.edu. 


\section{Authors}

Dennis Zaritsky, Helene M. Courtois, Juan Carlos Munoz-Mateos, Jenny Sorce, S. Erroz-Ferrer, S. Comeron, D. A. Gadotti, A. Gil de Paz, J. L. Hinz, E. Laurikainen, T. Kim, J. Laine, K. Menendez-Delmestre, T.

Mizusawa, M. W. Regan, H. Salo, M. Seibert, K. Sheth, E. Athanassoula, A. Bosma, M. Cisternas, Luis C. Ho, and Benne W. Holwerda

This article is available at ThinkIR: The University of Louisville's Institutional Repository: https://ir.library.louisville.edu/ 


\title{
THE BARYONIC TULLY-FISHER RELATIONSHIP FOR S ${ }^{4}$ G GALAXIES AND THE “CONDENSED” BARYON FRACTION OF GALAXIES
}

\author{
Dennis Zaritsky ${ }^{1}$, Helene Courtois ${ }^{2,3}$, Juan-Carlos Muñoz-Mateos ${ }^{4}$, Jenny Sorce ${ }^{2,5}$, S. Erroz-Ferrer ${ }^{6,7}$, \\ S. Comerón ${ }^{8,9}$, D. A. Gadotti ${ }^{10}$, A. Gil de Paz ${ }^{11}$, J. L. Hinz ${ }^{12}$, E. Laurikainen ${ }^{8,9}$, T. Kim ${ }^{4,10,13,14}$, J. Laine ${ }^{8}$, \\ K. MenéndeZ-Delmestre ${ }^{15}$, T. Mizusawa ${ }^{4,16}$, M. W. Regan ${ }^{17}$, H. Salo $^{8}$, M. Seibert ${ }^{14}$, K. Sheth $^{4}$, \\ E. Athanassoula ${ }^{18}$, A. Bosma ${ }^{18}$, M. Cisternas ${ }^{6,7}$, Luis C. Ho ${ }^{14,19}$, and B. Holwerda ${ }^{20}$ \\ ${ }^{1}$ Steward Observatory, University of Arizona, 933 North Cherry Avenue, Tucson, AZ 85721, USA; dennis.zaritsky@ gmail.com \\ ${ }^{2}$ Université Lyon 1, CNRS/IN2P3, Institut de Physique Nucléaire, Lyon, France \\ ${ }^{3}$ Institute for Astronomy, University of Hawaii, 2680 Woodlawn Drive, Honolulu, HI 96822, USA \\ ${ }^{4}$ National Radio Astronomy Observatory/NAASC, 520 Edgemont Road, Charlottesville, VA 22903, USA \\ ${ }^{5}$ University of Potsdam: Leibniz-Institut für Astrophysik, Potsdam, Germany \\ ${ }^{6}$ Instituto de Astrofísica de Canarias, Vía Láctea s/n 38205 La Laguna, Spain \\ ${ }^{7}$ Departamento de Astrofísica, Universidad de La Laguna, E-38206 La Laguna, Spain \\ ${ }^{8}$ Astronomy Division, Department of Physical Sciences, University of Oulu, P.O. Box 3000, FI-90014, Oulu, Finland \\ ${ }^{9}$ Finnish Centre of Astronomy with ESO (FINCA), University of Turku, Väisäläntie 20, FI-21500, Piikkiö, Finland \\ ${ }^{10}$ European Southern Observatory, Casilla 19001, Santiago 19, Chile \\ ${ }^{11}$ Departamento de Astrofísica, Universidad Complutense de Madrid, E-28040 Madrid, Spain \\ ${ }^{12}$ MMTO, University of Arizona, 933 North Cherry Avenue, Tucson, AZ 85721, USA \\ ${ }^{13}$ Astronomy Program, Department of Physics and Astronomy, Seoul National University, Seoul 151-742, Korea \\ 14 The Observatories of the Carnegie Institution of Washington, 813 Santa Barbara Street, Pasadena, CA 91101, USA \\ ${ }^{15}$ Universidade Federal do Rio de Janeiro, Observatório do Valongo, Ladeira Pedro Antônio, 43, CEP 20080-090, Rio de Janeiro, Brazil \\ ${ }^{16}$ Florida Institute of Technology, Melbourne, FL 32901, USA \\ ${ }^{17}$ Space Telescope Science Institute, 3700 San Martin Drive, Baltimore, MD 21218, USA \\ ${ }^{18}$ Aix Marseille Université, CNRS, LAM (Laboratoire d'Astrophysique de Marseille) UMR 7326, F-13388, Marseille, France \\ ${ }^{19}$ Kavli Institute for Astronomy and Astrophysics, Peking University, Beijing 100871, China \\ ${ }^{20}$ European Space Agency Research Fellow (ESTEC), Keplerlaan, 1, 2200 AG Noordwijk, The Netherlands \\ Received 2014 January 6; accepted 2014 February 4; published 2014 May 2
}

\begin{abstract}
We combine data from the Spitzer Survey for Stellar Structure in Galaxies, a recently calibrated empirical stellar mass estimator from Eskew et al., and an extensive database of $\mathrm{H}$ I spectral line profiles to examine the baryonic Tully-Fisher (BTF) relation. We find (1) that the BTF has lower scatter than the classic Tully-Fisher (TF) relation and is better described as a linear relationship, confirming similar previous results, (2) that the inclusion of a radial scale in the BTF decreases the scatter but only modestly, as seen previously for the TF relation, and (3) that the slope of the BTF, which we find to be $3.5 \pm 0.2\left(\Delta \log M_{\text {baryon }} / \Delta \log v_{c}\right)$, implies that on average a nearly constant fraction $(\sim 0.4)$ of all baryons expected to be in a halo are "condensed" onto the central region of rotationally supported galaxies. The condensed baryon fraction, $M_{\text {baryon }} / M_{\text {total }}$, is, to our measurement precision, nearly independent of galaxy circular velocity (our sample spans circular velocities, $v_{c}$, between 60 and $250 \mathrm{~km} \mathrm{~s}^{-1}$, but is extended to $v_{c} \sim 10 \mathrm{~km} \mathrm{~s}^{-1}$ using data from the literature). The observed galaxy-to-galaxy scatter in this fraction is generally $\leqslant$ a factor of 2 despite fairly liberal selection criteria. These results imply that cooling and heating processes, such as cold versus hot accretion, mass loss due to stellar winds, and active galactic nucleus driven feedback, to the degree that they affect the global galactic properties involved in the BTF, are independent of halo mass for galaxies with $10<v_{c}<250 \mathrm{~km} \mathrm{~s}^{-1}$ and typically introduce no more than a factor of two range in the resulting $M_{\text {baryon }} / M_{\text {total }}$. Recent simulations by Aumer et al. of a small sample of disk galaxies are in excellent agreement with our data, suggesting that current simulations are capable of reproducing the global properties of individual disk galaxies. More detailed comparison to models using the BTF holds great promise, but awaits improved determinations of the stellar masses.
\end{abstract}

Key words: galaxies: evolution - galaxies: formation - galaxies: fundamental parameters - galaxies: stellar content - galaxies: structure

Online-only material: color figures, machine-readable and VO tables

\section{INTRODUCTION}

Empirical galaxy scaling relations are a testament to the existence of underlying physical principles of galaxy formation. Among such scaling relations, the relationship between the width of the neutral hydrogen line and the luminosity of a galaxy (Tully \& Fisher 1977) stands as one of the most useful (for example, see its use as a distance estimator; Freedman et al. 2001) and constraining (for example, see its use to test complicated baryonic physics in dark matter halos; Steinmetz \& Navarro 1999).
Even though the Tully-Fisher relationship (hereafter the TF relation) has been extensively vetted and explored (Freedman \& Madore 2010), some questions remain unanswered. Foremost are those relating to the physical origin of the relationship. Although vaguely related to the virial theorem, the relationship is not simply a recasting of that theorem (cf. McGaugh \& de Blok 1998; McGaugh et al. 2000; Zaritsky 2012). In particular, one can imagine constructing two galaxies with the same rotation curve, but extending the stellar disk a few times farther out in one. Although both galaxies would satisfy the virial theorem, the two galaxies could not lie on the same relationship between 
rotation velocity and luminosity. Nature, apparently, cannot envision two such galaxies.

However, the TF relation in its simplest incarnation is not a complete description of all disk galaxies. It fails to match the characteristics of some faint, gas-rich galaxies (Carignan \& Beaulieu 1989; Persic \& Salucci 1991; Meurer et al. 1996; McGaugh et al. 2000). A scaling relationship is recovered if one recasts it as one between rotation velocity and baryonic mass rather than just luminosity (Freeman 1999; Walker 1999; McGaugh et al. 2000; Verheijen 2001; Geha et al. 2006), and this is referred to as the baryonic TF (hereafter, BTF). These results suggest that the original $\mathrm{TF}$ exists because for most galaxies in TF studies the luminosity is a reasonably precise proxy for stellar mass and the gas mass is negligible.

In a quest to uncover more clues and improve the fidelity of the TF as a distance estimator, investigators have long sought additional parameters that would help reduce the scatter in the TF relation - a search for a second, or even third, parameter beyond the rotational velocity (see, for examples, Strauss \& Willick 1995; Zwaan et al. 1995; Sprayberry et al. 1995; Courteau 1997; Courteau \& Rix 1999; McGaugh 2005b; Kassin et al. 2007; Hall et al. 2012). In this context, evidence for the importance of a scaling radius has been presented (cf. Yegorova \& Salucci 2007; Kassin et al. 2007; Hall et al. 2012), although many other studies found no such dependence (Zwaan et al. 1995; Sprayberry et al. 1995; Courteau \& Rix 1999; McGaugh 2005b).

The principal scaling relationship for early-type galaxies, the Fundamental Plane (Djorgovski \& Davis 1987; Dressler et al. 1987), includes a kinematic term, a scaling radius, and a surface brightness term. Following along that line of reasoning, we have explored a joint scaling relationship for both late and early types that has the flavor of the Fundamental Plane (Zaritsky et al. 2006a, 2006b, 2008; Zaritsky 2012). Given its antecedent and geometry, it is referred to as the Fundamental Manifold. The implication of that work in the current context is that disk galaxies should show residuals from the standard TF that correlate with the half light radius. In this study, we examine whether the introduction of this scaling radius improves the BTF as well.

Finally, the BTF has great promise to provide detailed, quantitative tests of galaxy formation and evolution models (for some examples of this approach, see Mayer \& Moore 2004; Gnedin et al. 2007; Governato et al. 2007; Avila-Reese et al. 2008; Gurovich et al. 2010; McGaugh 2012; Aumer et al. 2013). The limiting uncertainty in such tests has been the stellar mass determination. While prescriptions for the mass-to-light ratio based on observed colors are available (Bell \& de Jong 2001), these depend sensitively, at a level of precision that incapacitates the envisioned test, on the adopted stellar initial mass function (IMF) and star formation history. Because these techniques are based on stellar population modeling they also carry fundamental uncertainties on the modeling of rare, but luminous, phases of stellar evolution (Maraston et al. 2006; Conroy et al. 2009). As an alternative, Eskew et al. (2012) used the resolved stellar population study of the Large Magellanic Cloud (Harris \& Zaritsky 2009) and Spitzer images (Meixner et al. 2006) to calibrate the conversion of 3.6 and $4.5 \mu \mathrm{m}$ luminosities to stellar mass. This approach bypasses some, but not all, of the weaknesses mentioned above (see Eskew et al. 2012 for a discussion of the relative merits). The use of the IR tracers also mitigates the role of internal extinction and detailed work at these wavelengths aims to correct for dust emission as well (Meidt et al. 2012). With the advent of this new stellar mass estimator and large samples of galaxies observed with the Spitzer Space Telescope, we now return to reexamine the BTF.

In this study we combine homogeneous, high-quality IR data (3.6 $\mu \mathrm{m}$ and $4.5 \mu \mathrm{m}$ surface photometry) from the Spitzer Survey for Stellar Structure in Galaxies ( $S^{4} \mathrm{G}$; Sheth et al. 2010), which is less susceptible to extinction than optical data and has a more uniform stellar mass-to-light ratio, the Eskew et al. (2012) stellar mass estimator, and H I spectral line profiles from the literature curated by the Cosmic Flows project (Courtois et al. 2011) to re-explore some of the questions raised by both the standard and BTF relationship. A strength of this study is that the parent sample is fairly broadly selected to be a magnitude limited, volume limited sample. As such, the results are representative of galaxies in general rather than of a "pristine" sample intended to provide the tightest scaling relation or most reliable distances. In Section 2 we describe the data, present our findings regarding the BTF and the role of other parameters in Section 3, and conclude in Section 4.

\section{THE DATA AND MEASUREMENTS}

The photometric data, from which we obtain the measurements of the infrared luminosity, half light radii, and inclinations come from the $S^{4} G$ data set (Sheth et al. 2010) and subsequent analysis described by J.-C. Muñoz-Mateos et al. (2013, in preparation) and H. Salo et al. (2014, in preparation). The basic data consist of images obtained with the Spitzer Space Telescope (Werner et al. 2004) with the IRAC instrument (Fazio et al. 2004) during its warm mission, so limited to the $3.6 \mu \mathrm{m}$ and $4.5 \mu \mathrm{m}$ channels, of 2352 galaxies in the local universe. The data processing, masking, and photometry are described by J.-C. Muñoz-Mateos et al. (2013, in preparation), with additional model fitting described by H. Salo et al. (2014, in preparation). The data have been used in a variety of studies that can ultimately complement that of scaling relations, including those of Buta et al. (2010), Comerón et al. (2012), Holwerda et al. (2014), and Zaritsky et al. (2013).

Since 2009, the Cosmic Flows project (CF) has gathered all the digital H i spectra available from the public archives of the largest radio-telescopes worldwide and re-measured them in a consistent way. Two sub-projects of $\mathrm{CF}$, at Green Bank in the USA and at Parkes in Australia (Courtois et al. 2011), complete the archives for targets without previous observations that are adequate for TF studies.

The main goal of CF is to map the all-sky peculiar velocity field at redshift zero and reconstruct the underlying dark matter distribution. For that purpose, tens of thousand of galaxy line widths were measured with a new robust method described by Courtois et al. (2009, 2011). Briefly, the line width parameter, $W_{m 50}$, is a measure of the $\mathrm{HI}_{\mathrm{I}}$ profile width at $50 \%$ of a specially calculated estimate of the maximum flux within the velocity range encompassing $90 \%$ of the total $\mathrm{H}$ I flux (details provided in the cited references and computer code available from H. Courtois). This measurement is then transformed into the parameter $W_{m x}^{a v}$ by correcting for the slight relativistic broadening, broadening due to finite spectral resolution, internal turbulent motions (see Equation (2) in Courtois et al. 2011), and averaged if there are multiple good measurements. Further details can be found in Courtois et al. $(2009,2011)$ and Tully $\&$ Courtois (2012). The question of whether one should use a direct measurement of the maximum of the rotation curve, the width of the H I profile as a proxy for that maximum velocity, or the rotational velocity measured over the flattest part of the 

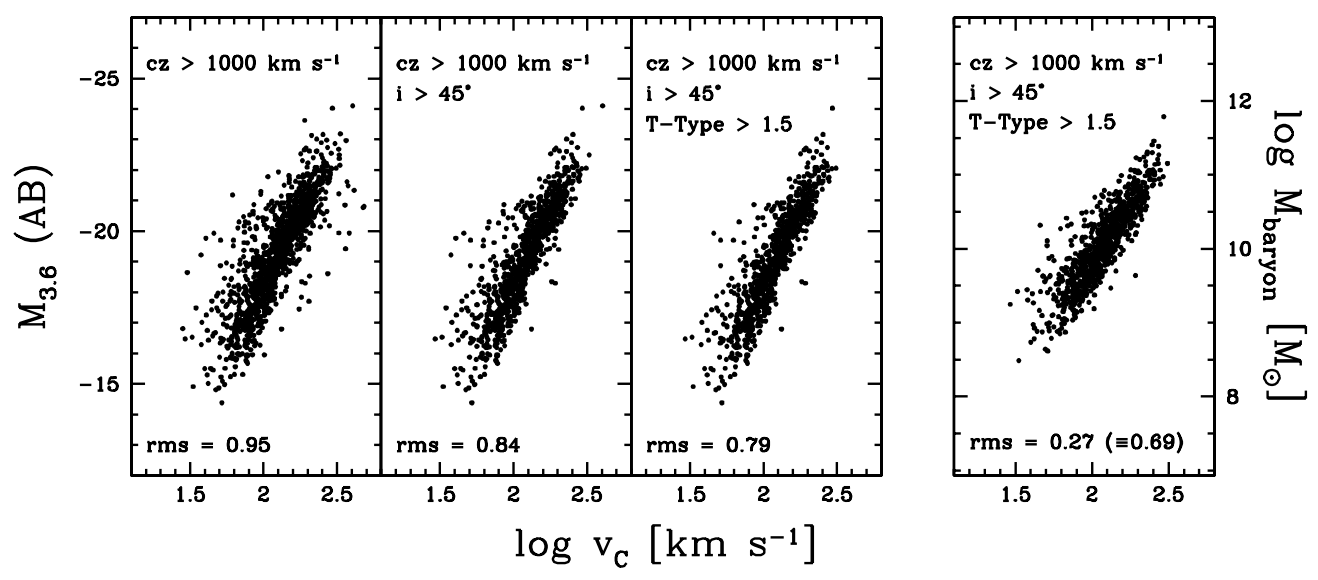

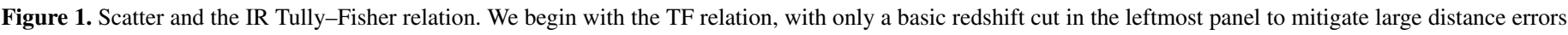

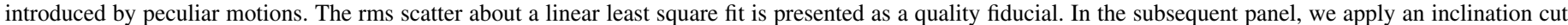

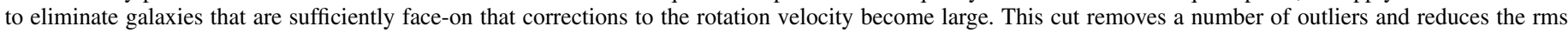

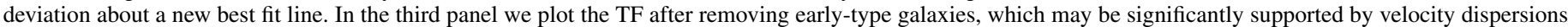

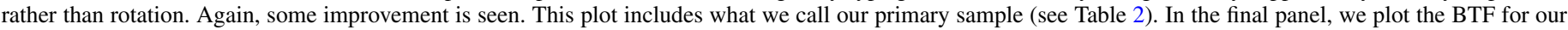

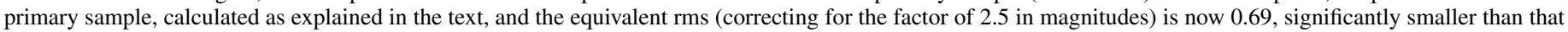
for the TF of the same sample.

rotation curve is a long-standing one in TF work that we do not have the data to address. Given the data available to us, we use the width of the H I profile, and in particular utilize $W_{m 50}$, which has been shown to be superior to alternative parameterizations of the line width (Courtois et al. 2009).

We currently have coherent H I measurements for 16,121 galaxies. Of those, 12,189 are of sufficient quality for distance measurements with TF. This catalog is available for public use at the Extragalactic Distance Database Web site ${ }^{21}$ and we call it the "All Digital H i catalog." Several other parameters available are included (Tully et al. 2009) such as the integrated H I line fluxes computed from the $\mathrm{H}$ I lines, which have a flux calibration uncertainty of about $10 \%-15 \%$, and the average heliocentric velocities.

To calculate the TF parameters, we define our maximum circulate velocity, $v_{c}$, as $v_{c}=W_{m x}^{a v} /(2 \sin i)$, where $i$ is the inclination. We adopt inclinations from our analysis of the $S^{4} \mathrm{G}$ images (H. Salo et al. 2014, in preparation), which includes multi-component galaxy modeling and produces an inclination measurement for the disk component. We find that the TF residuals using this inclination are smaller than those using $B$-band inclinations from RC3 (deVaucouleurs et al. 1991), a photometric band that emphasizes the disk over the bulge. Nevertheless, this approach, like any that use photometric measurements to estimate inclination and gaseous kinematics to measure the rotation velocities, implicitly assumes that the stars and gas are coplanar and that the stellar isophotes are intrinsically round.

One can then derive a distance using

$$
\log D_{L}=\left(m_{3.6}+20.34+9.74\left(\log v_{c}-2.5\right)-25\right) / 5,
$$

which comes from a recent IR TF calibration (Sorce et al. 2013), where $m_{3.6}$ is the total apparent magnitude derived in the Spitzer photometric band at $3.6 \mu \mathrm{m}$. However, here we use the smooth Hubble flow distances, calculated from the CMB-frame recessional velocities, to avoid circularity when examining the TF residuals. We adopt $\mathrm{H}_{0}=70 \mathrm{~km} \mathrm{~s}^{-1} \mathrm{Mpc}^{-1}, \Omega_{m}=0.3$, and $\Omega_{\Lambda}=0.7$ and account for the differences between luminosity

\footnotetext{
21 http://edd.ifa.hawaii.edu; catalog "All Digital H I"
}

and angular distances, the latter used for our calculation of radii. Finally, for our calculation of the BTF we use the H I flux parameter to compute the H I mass of a given galaxy using the equation

$$
M_{\mathrm{HI}}=2.36 \times 100000 \times D_{L}^{2} \times F
$$

in units of $10^{5} M_{\odot}$, where $M_{\mathrm{H} \text { I }}$ is the $\mathrm{HI}_{\mathrm{I}}$ gas mass, $D_{L}$ is the luminosity distance in $\mathrm{Mpc}$, and $F$ is the flux integrated within the $\mathrm{H}$ I line profile in units of $\mathrm{Jy} \mathrm{km} \mathrm{s}^{-1}$. Together, we have 1468 galaxies for which we have the necessary $\mathrm{S}^{4} \mathrm{G}$ and $\mathrm{H}$ I data.

\section{RESULTS}

\subsection{The $S^{4} G$ TF Relation and Sample Selection}

The basic TF relation for $\mathrm{S}^{4} \mathrm{G}$ galaxies is shown in the leftmost panel of Figure 1, with the only exclusion being galaxies that have recessional velocities that are less than $1000 \mathrm{~km} \mathrm{~s}^{-1}$. This criterion removes 218 galaxies from consideration. The recessional velocity cut is the lowest possible to avoid including galaxies whose peculiar velocities, which can range as high as a few hundred $\mathrm{km} \mathrm{s}^{-1}$ except in the centers of galaxy clusters where they can be larger, introduce significant errors in the inferred distances. We will show later that this cut needs to be significantly increased.

The goal of this study is not to present the functional form of an optimal IR TF relation (see Sorce et al. 2013 for that line of inquiry), but we do want to compare the scatter for different incarnations of the scaling relations. Therefore, we fit a line using least squares to provide a fiducial against which to compare the scatter and quote the rms about that fit in the lower left of the panel. The existence of a basic TF relation is evident, although there is obvious scatter. Whether this scatter is reducible either through the exclusion of galaxies that are illsuited for this measurement, or the inclusion of, or correction for, another physical parameter in the scaling relation, or whether it is irreducible observational noise is what we now explore.

We have already made one defensible selection cut on recessional velocity, and we next explore one on disk inclination relative to the line of sight. Because the measured rotational velocity must be corrected for inclination, as the disks become 
Table 1

Primary Sample

\begin{tabular}{|c|c|c|c|c|c|c|c|c|c|}
\hline PGC No. & Alternative Name & $\begin{array}{c}\log v_{c} \\
\left(\mathrm{~km} \mathrm{~s}^{-1}\right)\end{array}$ & $\begin{array}{c}\log M_{\text {baryon }} \\
\left(M_{\odot}\right)\end{array}$ & $\begin{array}{c}\log M_{*} \\
\left(M_{\odot}\right) \\
\end{array}$ & $\begin{array}{c}\log M_{\text {atomic }} \\
\left(M_{\odot}\right)\end{array}$ & $\begin{array}{c}\log M_{\mathrm{mol}} \\
\left(M_{\odot}\right)\end{array}$ & $\begin{array}{c}i \\
\left({ }^{\circ}\right)\end{array}$ & T-type & $\begin{array}{c}c z \\
\left(\mathrm{~km} \mathrm{~s}^{-1}\right)\end{array}$ \\
\hline 72957 & ESO012-010 & 2.07 & 10.01 & 9.48 & 9.81 & 8.77 & 62 & 7.7 & 1925 \\
\hline 181 & ESO012-014 & 1.72 & 9.87 & 9.17 & 9.78 & -9.99 & 65 & 9.0 & 1936 \\
\hline 13695 & ESO015-001 & 1.82 & 9.72 & 8.96 & 9.63 & -9.99 & 65 & 9.6 & 1659 \\
\hline 13931 & ESO054-021 & 2.04 & 10.13 & 9.73 & 9.87 & 8.68 & 60 & 7.9 & 1424 \\
\hline 2445 & ESO079-005 & 1.96 & 9.82 & 9.28 & 9.59 & 8.90 & 53 & 7.0 & 1599 \\
\hline 16780 & ESO085-047 & 1.51 & 9.42 & 8.74 & 9.32 & -9.99 & 57 & 9.0 & 1491 \\
\hline 18051 & ESO120-021 & 1.81 & 9.32 & 8.43 & 9.26 & -9.99 & 60 & 10.0 & 1364 \\
\hline
\end{tabular}

(This table is available in its entirety in machine-readable and Virtual Observatory (VO) forms in the online journal. A portion is shown here for guidance regarding its form and content.)

more face on this correction becomes larger and uncertainties in the inclination measurement dominate. Given the large size of the sample, we can afford to be conservative and ignore systems that are relatively face-on, thereby avoiding this problem entirely. We have selected to include only those galaxies with inclinations $>45^{\circ}$. We will return to justify this specific selection below (Section 3.2). This cut removes an additional 278 galaxies from consideration. Optical studies also face problems at large inclination because of the required high extinction corrections to the total magnitude. By observing at $3.6 \mu \mathrm{m}$, we find that this problem is significantly reduced, as we will show later (Section 3.2). The result of our lower bound on inclination is seen in the second panel in Figure 1. A number of outliers are removed and the rms scatter is reduced.

Next, we consider that the standard TF relation does not describe early-type galaxies, which can have stellar components with almost no rotation that are quite luminous. To eliminate these galaxies, we consider only galaxies of T-type $>1.5$ (Sab's and later). Again, this helps reduce the scatter as can be seen in the third panel of Figure 1 and reduces the sample by a further 79 galaxies to a total of 903 galaxies. This set of galaxies is what we refer to as our primary sample.

Despite the improvements that these criteria have realized, there are at least two problems they have not fully addressed. First, the scatter upward from the ridge line remains significant. It is curious, and perhaps telling, that the lower edge of the ridgeline is particularly sharp and well-defined, suggesting that random observational errors are not the dominant cause of the remaining scatter. Second, the relationship is not a straight line but rather has a downward kink at $\log v_{c} \sim 2$. This is exactly the class of feature that has been seen in studies advocating the BTF over the classic TF, particularly for low-luminosity galaxies (McGaugh et al. 2000).

\subsection{The Baryonic TF Relation}

Following that line of reasoning, we now calculate the baryonic mass of these galaxies. The stellar mass we obtain using the IR calibration of Eskew et al. (2012) based on spatially resolved stellar population studies of the Large Magellanic Cloud (Harris \& Zaritsky 2009; Meixner et al. 2006) that enables us to convert the combination of 3.6 and $4.5 \mu \mathrm{m}$ fluxes to a corresponding number of solar masses $\left(M_{*}=10^{5.65} F_{36}^{2.85} F_{45}^{-1.85}\right)$. This estimator has subsequently been confirmed both with comparison to Sloan Digital Sky Survey-derived stellar masses (Cybulski et al. 2014) and with detailed dust/star decompositions of the IR flux (M. Querejeta et al. 2014, in preparation). To estimate the gas mass we use the relationship described above to obtain $M_{\mathrm{H}}$, adopt a sliding scale in the correction for the $\mathrm{H}_{2}$ mass as a function of galaxy type (Young \& Knezek 1989; Young \& Scoville 1991), and correct for the mass in He and metals by multiplying by 1.4 . We adopt the parameterization of the dependence of molecular mass fraction with galaxy type presented by McGaugh \& de Blok (1997): $M_{\mathrm{H}_{2}} / M_{\mathrm{H}_{\mathrm{I}}}=3.7-0.8 T+0.043 T^{2}$, where $T$ is the galaxy T-type. In a small number of cases this formula results in an unphysical value (negative, but small) for the molecular mass and in those cases we set it to 0 . The correction for the molecular gas mass is often ignored because it is negligible in the relevant class of galaxy (see Geha et al. 2006 for one such example). We do include it, but find that it makes only a modest difference for our sample. Alternative prescriptions, based, for example, on the surface density of $\mathrm{H}$ I, also exist (Leroy et al. 2008; McGaugh 2012), but we do not have the necessary information to apply this approach and the estimation of the molecular mass is a minor source of uncertainty here. The resulting mass estimates, and other parameters necessary for the BTF, are presented in Table 1 for our primary sample.

We refer to the sum of the stellar and gaseous mass calculated in this manner as the baryonic mass, $M_{\text {baryon, and plot it as }}$ a function of $v_{c}$ in the rightmost panel of Figure 1. It is important to note, however, that despite the common usage of the term baryonic mass in this context we have not included the potential contributions of either extremely cold material (few K) that evades the molecular measurements (see, for examples, Pfenniger \& Revaz 2005 and references therein) or warm $\left(>10^{5} \mathrm{~K}\right)$ gas that may be present. Furthermore, we have not included the presence of baryonic material at large radii. Evidence for extended distributions of stars has been presented in early-types (Tal \& van Dokkum 2011), for extended star formation as a general feature in a significant fraction of latetypes (Thilker et al. 2005; Gil de Paz et al. 2005; Zaritsky \& Christlein 2007; Herbert-Fort et al. 2012), and for dust at large radii (Zaritsky 1994; Nelson et al. 1998; Ménard et al. 2010). We do not, therefore, expect this to be the full baryon accounting of galaxies. Instead, this represents the fraction of baryons that have "condensed" onto the central parts of galaxies.

Examining the BTF, we find that once again the scatter has improved (to an equivalent scatter of 0.69 after correcting for the factor of 2.5 present in the magnitudes that is not present in the $\log$ (masses)) and that this improvement comes not from removing outliers but from straightening the "kink" seen in the previous panels at $\log v_{c} \sim 2$. It is evident that the BTF, with nearby, face-on, and early-type galaxies removed, is the tightest scaling relation of those examined so far. For distance work, we 


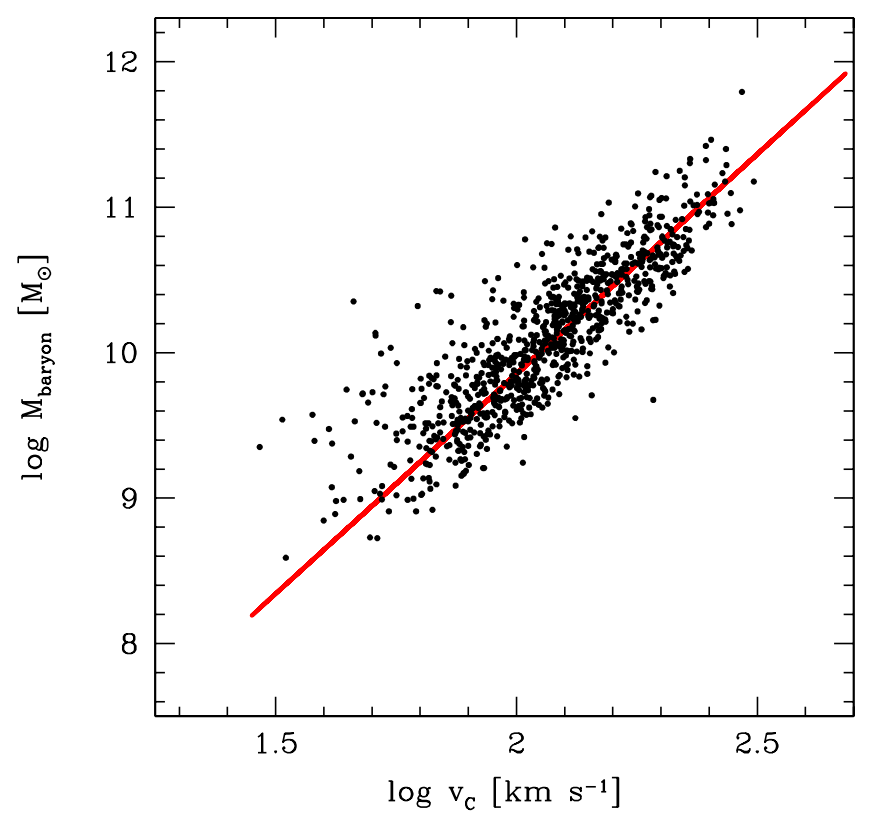

Figure 2. BTF for our primary sample (see Table 2). The line represents our fiducial about which we calculate residuals, derived using a bisector regression fit (Isobe et al. 1990). The fitted line for galaxies with $\log v_{c}>1.7$ has slope $3.022 \pm 0.007$, but the internal uncertainty is a gross underestimate of the true uncertainty.

(A color version of this figure is available in the online journal.)

recommend using the $\mathrm{BTF}$ if possible, particularly if the sample includes galaxies with $v_{c}<125 \mathrm{~km} \mathrm{~s}^{-1}$. However, the classic TF can show remarkably small scatter in appropriately selected samples. Verheijen (2001) finds that in a sample of galaxies in the Ursa Major cluster there is no evidence for any intrinsic scatter. For some lines of inquiry, a well-selected sample and exquisite data provide unique insights. Here, however, we are considering the broader disk galaxy population, warts and all.

Despite the significant improvements obtained in the scaling relations described above, the upward scatter of points remains (Figure 2). The asymmetric nature of that scatter again suggests that random observational errors are not solely at work. Historically, studies of TF and other scaling relations have made a number of difficult-to-reproduce selection cuts to clean the samples of such outliers, for example, often based on a galaxy's visual appearance or on the nature of the H i spectral line profile. Such culling may indeed be appropriate for certain work, such as a determination of $\mathrm{H}_{0}$, but are more questionable for comparison to models of galaxy evolution where the whole range of the population needs to be explored. This line of reasoning is particularly important when comparing to simulations, where it is difficult to control for different influences particularly because one does not know which ones are critical. For example, while requiring that a galaxy not suffer a major merger for $z<1$ may ensure that the simulated galaxy contains a thin disk there is no guarantee that it will match specific visual criteria for interaction signatures or deviations from a classic double-horn $\mathrm{H}$ i spectral line profile. Current high-resolution simulations (e.g., Aumer et al. 2013) are limited in the number of galaxies simulated, so they do attempt to target a constrained class of galaxy, such as those with a thin disk, but the resulting relation to empirical criteria is uncertain. On the observational side there is therefore a delicate balance in determining which selection criteria are well motivated in an effort to recover the intrinsic scatter in a scaling relation and which may artificially reduce the scatter. We now

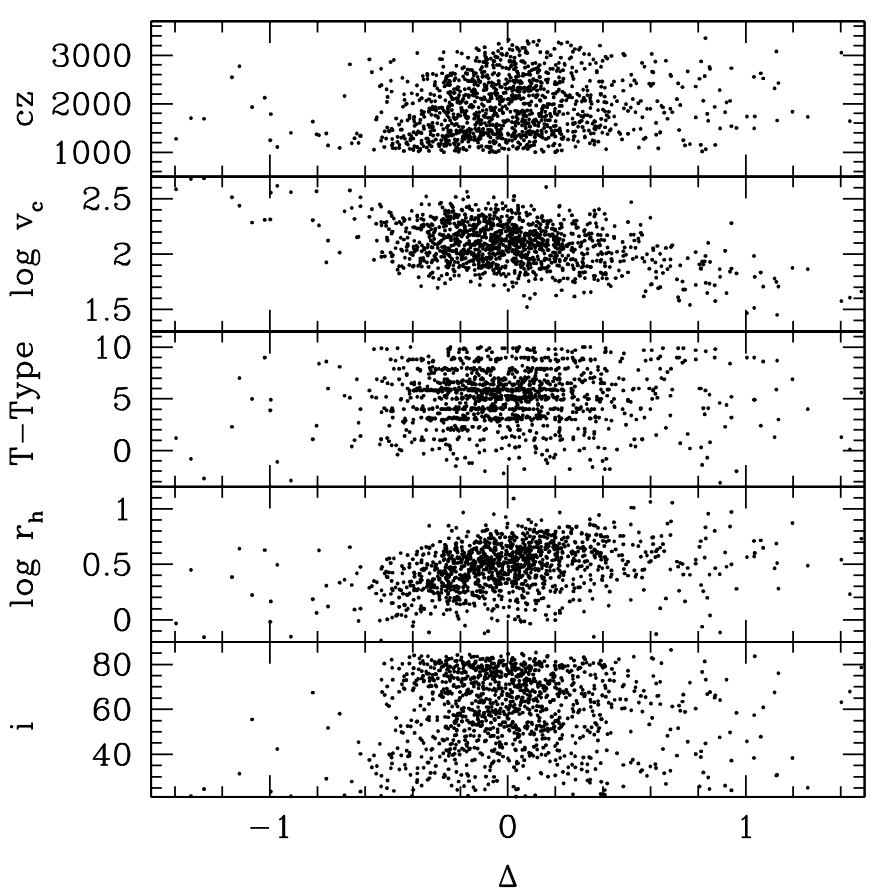

Figure 3. Residuals from the BTF and various galaxy parameters. Here we plot our complete sample of 1478 galaxies (a few lie off the plot boundaries). The units on inclination, $i$, are degrees, on $r_{h} \mathrm{kpc}$, and on $v_{c}$ and $c z \mathrm{~km} \mathrm{~s}^{-1}$.

discuss our approach at refining appropriate selection criteria or parameters in an effort to uncover a realistic scaling relation with the minimal scatter that outlines the true underlying relationship.

Beginning with the BTF relation, reproduced in Figure 2, we examine the nature of the residuals about a fitted line (slope $=$ $3.022 \pm 0.007$, for galaxies with $\log v_{c}>1.7$ to eliminate the tail of outliers). The fit is the result of a bisector regression fit (Isobe et al. 1990) and we will return to a discussion of choice of fitting method below because indeed the fitting algorithm can be a significant source of uncertainty in this type of work. Not only for this reason, the internal uncertainty is a gross underestimate of the true uncertainty. For now this line serves as a fiducial against which to calculate residuals. We plot the residuals about the line relative to various characteristics of the galaxies in Figure 3. We are in general looking for two broad classes of phenomena. First, we look for a significant increase in scatter over a limited parameter range. Such a feature suggests either that we cannot measure galaxy properties sufficiently well for this parameter range, for example, at low inclinations, or that the TF relationship is not applicable, for example, for early-type galaxies. We find both of these effects in Figure 3. Examining the panel showing the residuals, $\Delta$, relative to inclination, there is no concentration of galaxies about $\Delta=0$ for inclinations $<40^{\circ}$. This justifies our cut at $45^{\circ}$. Examining the panel showing the relation between $\Delta$ and T-type, there is no concentration toward $\Delta=0$ for T-type $<2$, supporting our criterion of T-type $>1.5$. Second, we look for a systematic trend in residuals suggesting either a systematic error or additional physical information. There are two fairly evident such trends in Figure 3. First, there is that of $\Delta$ with $v_{c}$, although that is mostly defined by long tails of high residuals. In the core of the distribution such a correlation is not so clear. Second, there is a trend of $\Delta$ with the half light radius, $r_{h}$, that is mostly present in the core of the distribution, but not in the tail of high residuals. We will examine these in 
Table 2

Sample Selection Criteria

\begin{tabular}{lcc}
\hline \hline & Primary Sample & Low Scatter Subsample \\
\hline Extant H i observation & Yes & Yes \\
Recessional velocity & $c z>1000 \mathrm{~km} \mathrm{~s}^{-1}$ & $c z>2000 \mathrm{~km} \mathrm{~s}^{-1}$ \\
Inclination & $45^{\circ}<i$ & $45^{\circ}<i<80^{\circ}$ \\
T-type & $1.5<T$ & $3<T<8$ \\
\hline
\end{tabular}

greater detail, once we have dealt with additional sources of scatter that we identify on the basis of this figure.

Two sources of scatter, low inclinations and early T-types, we have already addressed. We identify two additional sources of scatter that we have not yet mitigated. First, looking at the panel showing $\Delta$ versus $v_{c}$, and in particular the tail of large residuals, we see significantly larger scatter among galaxies with lower recessional velocities. We suspect that this result is caused by larger relative distance errors due to peculiar velocities for these less luminous galaxies, which in a flux limited sample tend to be nearer. On the other hand, without additional information, we cannot exclude that the larger scatter is due to greater intrinsic scatter among fainter galaxies. However, previous studies that focused on low luminosity galaxies found that they do follow the BTF with low scatter (Geha et al. 2006; McGaugh 2012), so we conclude that peculiar velocities are the likely culprit. We therefore raise our cut to include only galaxies with $c z>2000 \mathrm{~km} \mathrm{~s}^{-1}$ for a new "low scatter" subsample. Second, examining the panel that contains the T-types, we see that there is no concentration about $\Delta=0$ for the largest T-types. This can either be interpreted as a true physical failing of the scaling relation for these galaxies or as evidence that either the rotation velocities or inclinations have much larger uncertainties for these galaxies. Regardless of the origin of the scatter, we will exclude galaxies with T-type $\geqslant 8$ from our "low scatter" subsample. Lastly, there appears to be a slight offset in the mean residual for galaxies with inclinations close to $90^{\circ}$, perhaps as a result of high internal extinction. To be conservative, we also increase our lower bound on T-type to 3 and impose an upper inclination bound of $80^{\circ}$ for our low scatter subsample. The primary and low-scatter subsample criteria are reprised in Table 2 .

Implementing these additional cuts, we now return to the systematic behavior of the residuals with respect to $r_{h}$. In Figure 4 we show this behavior relative to the slope $=3$ line shown in Figure 2, and for slope 2.5 and 3.5 lines. Regardless of the fiducial against which we calculate the residuals, there is a relationship between that residual and $r_{h}$. Even for what is visually the weakest case, that using the slope $=3.5$ line (rightmost panel), a Spearman rank correlation coefficient concludes that the chance the points are randomly distributed with respect to $r_{h}$ is $<2 \times 10^{-5}$. We conclude that the scatter in the BTF will be reduced by introducing a dependence on $r_{h}$, and we return to this below. The new cuts also greatly reduce the high and low $\Delta$ tails in the $\log v_{c}-\Delta$ distribution.

Using the low-scatter subsample, we now examine the BTF and the effect of the inclusion of an $r_{h}$ term in Figure 5. Fitting a linear relationship between $\log M_{\text {baryon }}$ and $\log v_{c}$, using here an ordinary least-squares approach and rejecting galaxies that have residuals $>0.6 \mathrm{dex}$, results in a slope of 3.28 , and an rms about the fit of 0.189 . To include an $r_{h}$ dependence, we fit an equation of the form $\log M_{\text {baryon }}=A \log v_{c}+B \log r_{h}+C$, again excluding galaxies with residuals about the fit $>0.6$. The rms is only slightly lower at 0.184 . The gains achieved by

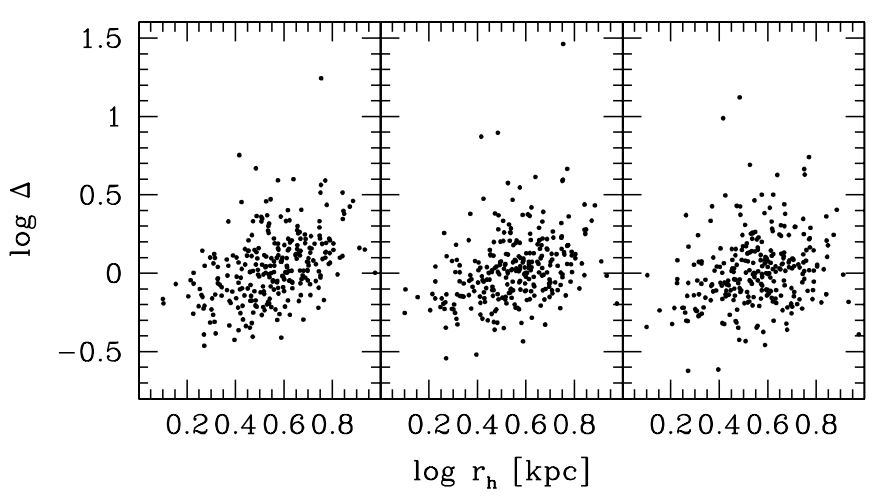

Figure 4. Residuals from the BTF and $r_{h}$ for our low scatter subsample (Table 2). We show the dependence of the residuals in the BTF with $r_{h}$ for three different choices of BTF slope. The central panel shows the residuals about a slope $=3$ line, while the left and right show the residuals about lines with slopes 2.5 and 3.5 , respectively. The choice of slope does affect the distribution of residuals, but even in the right panel, where the correlation between residual and $r_{h}$ is weakest, a Spearman rank correlation analysis indicates that there is a $1.5 \times 10^{-5}$ chance that these two quantities are unrelated.

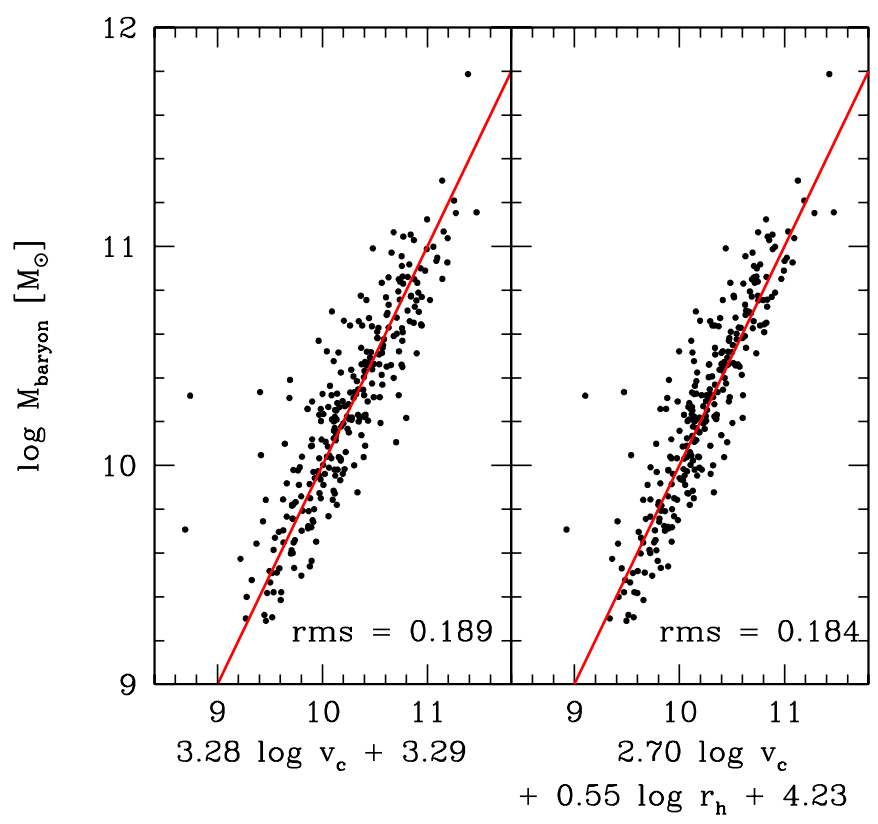

Figure 5. Comparison of fit vs. measured $M_{\text {baryon }}$ for BTF and scale-dependent BTF for our low scatter subsample. The plotted lines are the 1:1 relation. Improvement provided by including scale-dependence is noticeable but modest, and certainly not the primary source of the scatter.

(A color version of this figure is available in the online journal.)

including $r_{h}$ in the fit are detectable, but indeed modest. It is quite likely that the results of this analysis depend critically on the radius at which one measures the rotational velocity because of the sensitivity of the peak of the rotation curve on the degree of mass concentration and the lack of such sensitivity in the asymptotic value of the rotation curve. As such, our results apply to the use of $\mathrm{W}_{50}$ and are likely not directly applicable to other measurements of $v_{c}$ (see, for example, Verheijen 2001). We conclude that for disk galaxies over the present parameter range there is no pressing requirement to include an $r_{h}$ scaling and so continue our discussion with the standard BTF.

The scatter remains asymmetric, although there are now only a few outlying galaxies. Because we have removed the nearby galaxies that were susceptible to large distance errors, these outliers are probably due to dynamical effects, such 


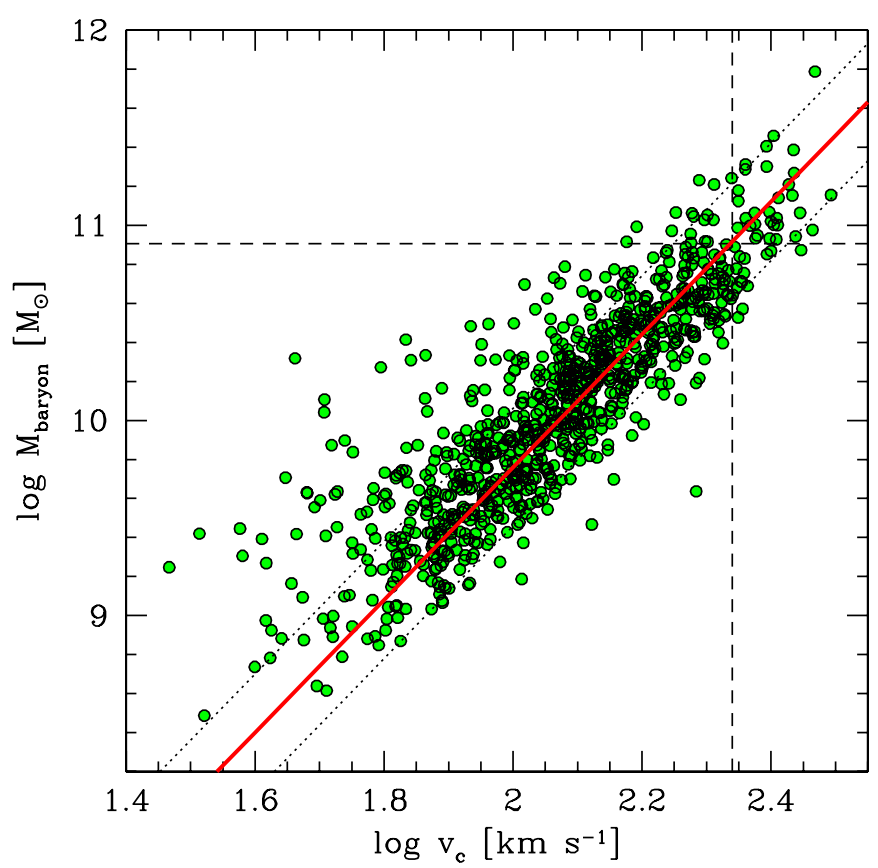

Figure 6. Baryonic Tully-Fisher compared to a simple model in which galaxies condense out $42 \%$ of their baryons as stars or cold gas (thick red line) and follow the halo mass $-v_{c}$ relation from Bullock et al. (2001). The dashed lines show the position of the MW that is used to provide a coarse normalization of the relationship. The inclined dotted lines show factors of two deviation from the model in the condensed baryon fraction. The simple description of a constant condensed baryon fraction is an excellent description of the sample mean, with variations of up to a factor two for individual galaxies allowed by the current level of scatter.

(A color version of this figure is available in the online journal.)

as interactions, that disturb the Hi velocity field (Ho 2007). Similar tails to the distribution have been noticed before (Ho 2007) and are not a unique artifact of our data. Indeed, careful pruning of samples based on interaction signatures, either in the morphology or the nature of the Hi spectral line profile often removes such outliers (Verheijen 2001). This population, if indeed the deviations are physically driven, should be included in comparisons to simulations because it is difficult to ensure that attempts to prune both the theoretical and empirical samples of "disturbed" galaxies will fairly and completely reproduce the selection.

\subsection{The "Condensed" Baryon Content of Galaxies}

As shown by our consideration of the $r_{h}$ term, the dominant term in the mass estimation is $v_{c}$. Therefore, we return to consider the BTF, reprised in Figure 6 using our primary sample. In the simplest model of galaxies we envision, the mass of a halo of characteristic velocity $v_{c}$ is proportional to $v_{c}^{3}$. Two powers of $v_{c}$ come from $M \propto v^{2} r$ and the third comes from the virial radius, $r$, having the characteristic that $r \propto v_{c}$, which is generically expected for dark matter halos (see Mo et al. 2010 for a review of this topic). If the fraction of baryons that settle, or condense, onto the central portion of the galaxy is a fixed number, then in this simple model we would expect a slope $=$ 3 line to fit the data as plotted. The normalization of that line would then provide the numerical value of that fixed fraction. In detail, when the growth of halos is simulated in a cosmological context the actual value of how halo mass depends on circular velocity differs slightly from our naive expectation ( slope $=3.4$; Bullock et al. 2001).
To compare the theoretical expectation to our data, we superpose the expectation on the data in Figure 6. Using the Milky Way (MW) as a reference, adopting $v_{c} \sim 220 \mathrm{~km} \mathrm{~s}^{-1}$ and total mass of $\sim 1.2 \times 10^{12} M_{\odot}$, an estimate that has remained relatively unchanged since observations of distant test particles became available (Zaritsky et al. 1989; Watkins et al. 2010; Boylan-Kolchin et al. 2013; Barber et al. 2014), we find that a galaxy with the total mass of the MW would have a baryonic mass, defined in the manner we have done so far in terms of the stellar mass plus 1.4 times the $\mathrm{H}$ I mass plus the correction for molecular gas, of $8.3 \times 10^{10} M_{\odot}$. When compared with the total mass, we derive a "condensed" baryon mass fraction $\left(M_{\text {baryon }} / M_{\text {total }}\right)$ of 0.07 . If we adopt that $M_{\text {total }} \propto v_{c}^{3.4}$, following Bullock et al. (2001), and posit that on average galaxies have the same condensed matter fraction, then we arrive at the solid red line in the figure. The concurrence, in the mean, is manifestly excellent.

The condensed baryon fraction we derive, 0.07 , should be compared with the universal value of the baryon to total matter ratio (0.1649 from WMAP9; Hinshaw et al. 2013), which then suggests that rotationally supported galaxies consistently condense out, either as stars or cold gas, about $40 \%$ of all of their baryons onto their central, luminous, regions regardless of their circular velocity, over the range of circular velocities shown in the figure. The $v_{c}$ range can be extended to lower values of $v_{c}$ with observations that have targeted low luminosity galaxies (McGaugh \& de Blok 1998; Geha et al. 2006; Trachternach et al. 2009; Stark et al. 2009) and the relationship still holds (see below). In other words, we find no evidence of significant differential baryonic mass loss within this sample, confirming previous work on the BTF (McGaugh 2005a; Geha et al. 2006; Trachternach et al. 2009; McGaugh 2012). The apparent consistency in this baryonic fraction is once again a testament to the regularity underlying galaxy formation and evolution, but is in tension with other estimates of the baryonic fraction (see end of Section 3.4).

The degree of scatter observed is significant, the dotted lines in Figure 6 represent a factor of two difference in either direction. The bulk of the galaxies lie within this factor of two range. The majority of the outliers well above this are removed once the cuts implemented for our low-scatter subsample are applied, and we explore the BTF for that sample below. The interesting question is how much of the scatter within the factor of two level is intrinsic. As we mentioned previously, some TF studies (Verheijen 2001) have found no evidence for intrinsic scatter. Our data are not suited to such an exploration because we do not have spatially resolved rotation curves, but we again stress that in terms of comparison to simulations a "pure" galaxy sample may not be the most appropriate comparison sample.

\subsection{Comparison to Previous Studies and Simulations}

The results on the condensed fraction and comparison to simulations are potentially highly constraining. McGaugh (2012) explore in detail comparisons to different theoretical predictions, both for $\Lambda C D M$ and non-standard (MOND) models. However, as he notes, a fundamental limitation of the current BTF is the determination of the stellar masses. In particular, the slope of the BTF can range between 3 and 4 depending on which photometric bands are used and significant variations are also possible depending on the fitting algorithm and treatment of outliers. Indeed, previous studies (Avila-Reese et al. 2008; Hall et al. 2012) have found shallower BTF slopes than that presented by McGaugh (2012), 4, and our use of a different stellar 
mass estimator results in data that are consistent with a BTF of slope 3.4 (Figure 6).

Stark et al. (2009) noted that one can avoid the uncertainties in the stellar modeling by studying galaxies in which the baryonic mass is dominated by the gas. Although determining the gas mass has its own issues (unknown molecular gas mass, corrections for metallicity), this approach provides a check on the results that are based on systems where stellar masses dominate. It does, however, assume that a single linear BTF applies to all rotationally supported galaxies. We exploit this idea by comparing the results for gas-dominated galaxies directly to those for stellar-dominated galaxies. By selecting only those galaxies in which one or the other component dominates, we separate the relative mass normalizations. We adopt the McGaugh (2012) measurements for galaxies in which the stellar mass contributes $<20 \%$ of the baryonic mass (these are typically low $v_{c}$ galaxies that are not in the $\mathrm{S}^{4} \mathrm{G}$ sample) and compare to those galaxies in our "low scatter" subsample that have gas masses that account for $<20 \%$ of the total baryonic mass.

This is not a perfect test. The McGaugh (2012) sample is treated differently than ours in a variety of ways. For example, the correction for metals is not applied (as these low mass galaxies have low metallicities) and resolved rotation curves are used to measure where the rotation curve is flat, $V_{f}$. Nevertheless, we can explore how each sample behaves independently as well as in combination. One factor we can correct for is the possibility of offsets in the stellar masses as calculated by McGaugh (2012) and ourselves, to ensure that the two galaxy samples are on the same stellar mass system (even those that are gas-dominated do have a stellar baryon component). For the eight galaxies in common to our samples, we find that on average $\log M_{*}$ differs by 0.215 , with the McGaugh (2012) masses being larger. We therefore "correct" our values upward to provide a direct comparison to his results in the left panel of Figure 7 and use these "corrected" masses when applying the criteria to select gas- and star-dominated galaxies. Using the same selected galaxies, but removing the "correction" factor (and so reducing the quoted McGaugh (2012) stellar masses), we obtain the results shown in the right panel of Figure 7. The two panels of the figure therefore comprise a test of the stellar mass prescriptions under the assumption that a single BTF applies across the full galaxy $v_{c}$ range.

There are various results of note in the figure. First, the fits to either the gas- or star-dominated galaxies in either panel result in fits that have nearly the same slope but different intercepts. Using the ordinary least-squares bisector regression fitting algorithm (Isobe et al. 1990), we find slopes of $3.32 \pm 0.19$ and $3.43 \pm 0.66$ for the stellar and gaseous samples when applying the correction to move our stellar mass estimates onto the McGaugh (2012) scale. Without the correction, we find slopes of $3.31 \pm 0.14$ and $3.43 \pm 0.66$, respectively. It would appear that the change in stellar mass normalization makes no significant difference and that in both cases the slopes for the gas- and star-dominated samples are similar. The lack of any effect on the slopes is a result of having selected gas- and star-dominated samples. In the former, changes in the stellar masses are nearly irrelevant and in the latter changes simply result in a zero-point shift of the stellar masses. This zero point change is evident in the result that between the corrected and uncorrected samples the intercept changes from 3.29 to 3.13 . The similarity in slopes also argues that other differences between the samples, such as the use of $V_{f}$ versus $\mathrm{W}_{50}$ or treatment of the gas masses, also have little

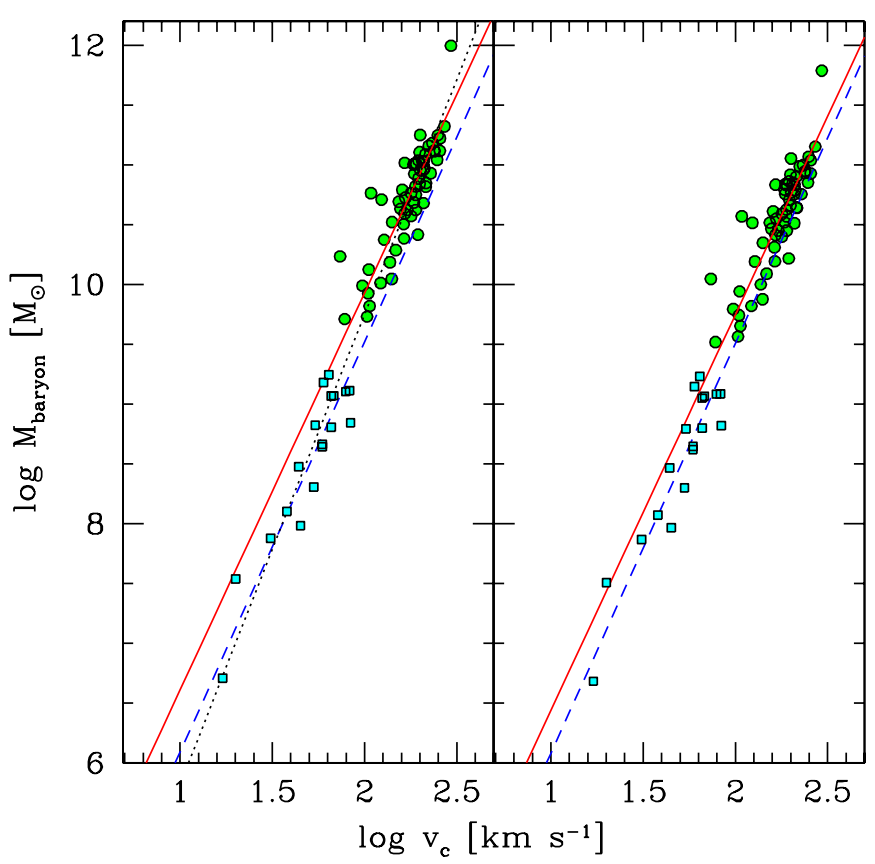

Figure 7. Baryonic Tully-Fisher for star-dominated galaxies (green circles and red solid line) compared to that for gas-dominated galaxies (cyan squares and blue dashed line). The data for gas dominated galaxies comes from McGaugh (2012). The left panel has our stellar masses renormalized to match on average those of McGaugh (2012) using a subset of galaxies in common to the two samples. In the right panel, we do not renormalize the stellar masses. The slopes of the fits done independently for the stellar- and gaseous-dominated samples are remarkably similar. The intercepts, however, vary. Because the intercepts vary less using our stellar mass estimates, we conclude that, under the assumption that a single, linear BTF is the correct underlying description of galaxies, our stellar mass estimates are to be preferred. The dotted line in the left panel, which is the bisector fit to the union of the two data sets results illustrates how fitting to the two samples together can result in a slope that is much larger than either sample suggests independently (3.93 \pm 0.19 in comparison to $3.32 \pm 0.19$ (stellar) and $3.43 \pm 0.66$ (gaseous)).

(A color version of this figure is available in the online journal.)

impact on the determination of the BTF slope. Nevertheless, an important next step would be to confirm this claim for a large sample of galaxies with both $V_{f}$ and $\mathrm{W}_{50}$. Second, all of these results are consistent with the slope $=3.4$ line plotted in Figure 6, suggesting good agreement with our theoretical expectations. Third, we demonstrate the importance of the stellar mass normalization when fitting a single relationship to the combination of the gas- and star-dominated samples. In the left panel of Figure 7 we also show this fit, which has slope $3.93 \pm 019$. While this fit appears to be a good description of the data, we know that it reflects the slope of neither the gas- or stardominated samples individually. In the case where we use our uncorrected stellar masses, the slope decreases to $3.68 \pm 0.17$ because the intercepts of our gas- and star-dominated galaxy fits are closer to each other. This result illustrates how one can obtain a much steeper BTF slope when combining gas- and star-dominated samples if the two have different intercepts (or mass normalizations). We stress that this demonstration does not necessarily invalidate the McGaugh (2012) results because we have not demonstrated such a failing within his self-consistent data set, it only highlights the importance the stellar mass normalization can have on BTF slope determinations.

One could take the argument further and advocate a larger reduction of the stellar masses to improve the agreement between the gas- and star-dominated galaxy fits. Such a reduction would 
not be unwarranted because the Eskew et al. (2012) relation is based on a Salpeter IMF. However, there are also uncertainties in the gas masses themselves, the sample is sufficiently sparse that the fitted parameters have large uncertainties, and the gas-dominated galaxies have not been measured in exactly the same manner as the $S^{4} \mathrm{G}$ sample. With larger samples of gas-dominated galaxies that are observed in a consistent manner with how the star-dominated ones are observed, it may be possible to obtain a more precise calibration of the stellar mass estimators, but such a treatment is currently premature.

Based on these results we conclude that our data favor a BTF slope between 3.3 and 3.7 , and so quote $3.5 \pm 0.2$. This is in agreement with some previous determinations (AvilaReese et al. 2008; Hall et al. 2012) and that our stellar mass normalization is a potential explanation for why our slope is in disagreement with others determinations (such as that of McGaugh 2012).

These results are all predicated on a particular choice of IMF, and on its universality. Recent results on early-type galaxies (Treu et al. 2010; van Dokkum \& Conroy 2010; Cappellari et al. 2012) and stellar clusters (Strader et al. 2011; Zaritsky et al. 2012, 2013), suggest that the IMF is not universal and that among galaxies the IMF variations track total mass. If such a pattern exists also among disk galaxies, then it will affect the BTF slope. To gauge the magnitude of the effect, we adopt the relationship between $M / L$ and velocity dispersion found by Cappellari et al. (2012), adopt $v_{c} / \sqrt{2.5} \sim \sigma$ (Burstein et al. 1997; Weiner et al. 2006; Zaritsky et al. 2006a; Zaritsky 2012), and recalculate the BTF slope using the low scatter subsample. We find a magnitude change in slope $<0.1$ (steeper), so subdominant to the current level of uncertainties arising from the cross calibration of the gas and star dominant galaxy samples. Eventually the BTF may also be a tool in addressing questions about IMF variations in disk galaxies, but greater precision is required.

Finally, we consider the most recent simulations that address where disk galaxies fall in the $M_{\text {baryon }}-v_{c}$ space (Aumer et al. 2013). Those simulations apply a multiphase smoothed particle hydrodynamics code with elaborate treatments of metal production, cooling rates, and metal diffusion to examine a range of galaxy properties. They include supernova feedback, but not active galactic nucleus feedback, and examine 16 simulated galaxies that range in halo mass from $10^{11}$ to $3 \times 10^{12} M_{\odot}$. In comparing to the McGaugh (2012) results they found some discrepancies that they attributed to details of their adopted prescriptions. However, comparing to our data (Figure 8), we find that their simulated galaxies do an excellent job of matching the properties of real galaxies. We conclude that current simulations are on track to reproduce the internal properties of galaxies, although the conflicting conclusions reached using either our or the McGaugh (2012) sample can also be taken as a cautionary tale regarding the importance of unresolved uncertainties in the stellar mass determinations.

We close by taking note of some tension between the BTF results, which indicate a constant $M_{\text {baryon }} / M_{\text {halo }}$ as a function of $M_{\text {halo }}$, and those obtained by comparing the statistical properties, such as number density and clustering, of galaxies to those of simulated dark matter halos (see Kauffmann et al. 1997; Benson et al. 2000; White et al. 2001; Berlind \& Weinberg 2002; Yang et al. 2004; Guo et al. 2010 and references therein for examples from a large set of pertinent references). Specifically, all studies based on statistical properties of galaxies and simulated or measured dark matter halo masses, the empirical masses

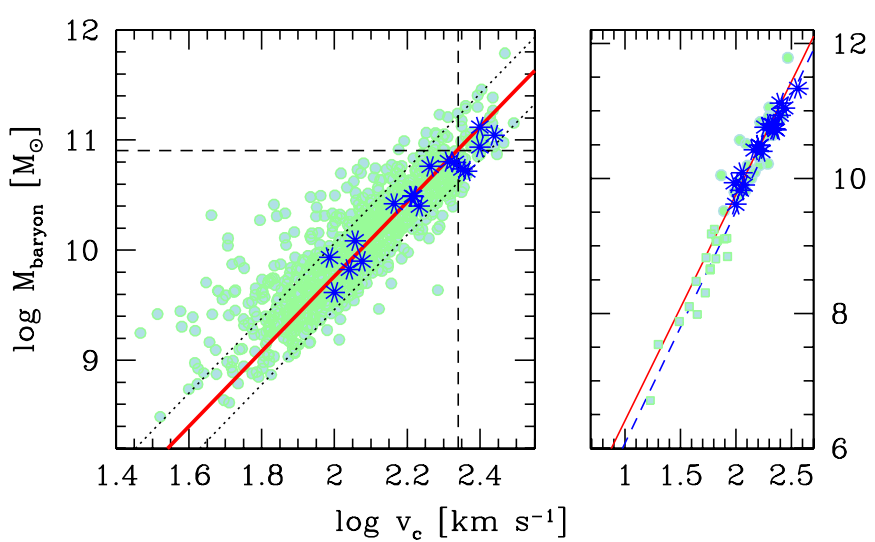

Figure 8. Comparison of simulated galaxies from Aumer et al. (2013) to our data. We reprise Figures 6 and 7 and superpose the simulated galaxies as blue stars. For the right panel we use our stellar masses (e.g., the right panel of Figure 7). The excellent agreement between the simulations and the data demonstrates how well current simulations are doing at reproducing the mean trend. The scatter is somewhat less than observed, but the simulations aimed to reproduce dynamically quiet disk galaxies. The various lines are the same as in the previous respective figures.

(A color version of this figure is available in the online journal.)

obtained via observations of gravitational lensing or satellite kinematics, indicate that the fraction of stellar mass, $M_{\text {stellar }}$, to dark matter mass, $M_{\text {halo }}$, peaks in galaxies with $M_{\text {halo }} \sim$ $10^{12} M_{\odot}$ and declines toward either more or less massive halos. TF and BTF have little to say on this issue at the large mass end, where rotationally supported galaxies are rare, but can inform results at the lower mass end. In fact, Guo et al. (2010) note that their results, based on the statistical approach, are also consistent with TF, which due to the kink we noted above supports a lower $M_{\text {stellar }} / M_{\text {halo }}$ for galaxies with low $v_{c}$. The declining stellar mass fraction in lower mass halos mass could be the result of either a lower efficiency of star formation or the loss of baryonic matter from which to form stars in these halos. Recent work applying the statistical treatment but relying not only on optical luminosity measurements, which trace the stars, but also including $\mathrm{H}$ I measurements suggest that that low mass galaxies retain a lower fraction of their baryons (Papastergis et al. 2012). This most recent result is in apparent conflict with the BTF results.

Both approaches purport to measure the baryon fraction and both have a history of consistent results that demonstrate their internal robustness. If we accept that each approach is free of unknown, and physically uninteresting, systematic errors, how can we resolve this conflict? One avenue might appeal to the fact that the results do not pertain to exactly the same systems, as the BTF results are for rotation-supported galaxies and the statistical ones are for the entire galaxy population and rotation supported galaxies become increasingly rarer at lower masses. Another approach might appeal to a higher degree of complexity in halo occupation models (cf. Zhu et al. 2006; Gao \& White 2007) even thought attempts to identify secondary factors beyond $M_{\text {halo }}$ have not identified such (see Tinker et al. 2008). Regardless of what the eventual resolution involves, the presence of even some galaxies with high $M_{\text {baryon }} / M_{\text {halo }}$ at low $M_{\text {halo }}$ demonstrates that the ability of a halo to retain its baryons is not solely related to $M_{\text {halo }}$, as is often envisaged in models that attempt to reproduce the decreasing $M_{\text {baryon }} / M_{\text {halo }}$ found by the statistical studies. Once again, rather than resolving the problem, we are only able to acknowledge the limits of our current understanding and urge caution in generalized treatments of galaxy formation and 
evolution, but also stress that simulations need to match both the statistical properties of galaxies and the individual global properties.

\section{CONCLUSIONS}

We examine the TF and BTF using the new sample of IR photometry provided by the $\mathrm{S}^{4} \mathrm{G}$ survey (Sheth et al. 2010; J.-C. Muñoz-Mateos et al. 2013, in preparation). The use of this large, homogenous sample brings the natural advantages of uniformity and large statistics, and also provides photometry that is less susceptible to reddening and stellar population variations. We apply a new empirical stellar mass estimator from Eskew et al. (2012) to obtain stellar masses for the sample. When combined with the available large, homogenized archival H I data of the Cosmic Flows program and complemented with additional observations (Courtois et al. 2011), we have a primary sample of 903 galaxies for study.

We confirm the superiority of the BTF for lower mass galaxies and that the overall scaling relation becomes noticeably more linear than the TF. Furthermore, we quantify the scatter introduced by various lax selection criteria related to the inclination limits of the sample, the morphological type range of the sample, and the effect of peculiar velocities on nearby galaxies. In the end, we are able to identify a BTF with a scatter of 0.18 dex, without detailed pruning of galaxies with either morphological or kinematic anomalies. This is a fair sample to compare to simulations where such cuts are difficult to reproduce.

We demonstrate that physical scale, here parameterized by the half light radius, correlates with the residual in the BTF. However, the gain in precision is exceedingly slight and the standard BTF $\left(\log M_{\text {baryon }}\right.$ versus $\left.\log v_{c}\right)$ is to be preferred for this galaxy sample for its simplicity.

We measure a BTF slope of $3.5 \pm 0.2$, consistent with the expectation where galaxies concentrate a fixed fraction of their initial baryons in the central, detectable components (stars and gas). Using the MW to normalize the relation, we show that the expectation of this simple model, where 0.07 of the total mass of the halo (or alternatively about $40 \%$ of the baryons) is "condensed" onto the central regions of the halo, is an excellent fit to the data. This agreement demonstrates that, independent of circular velocities for the range of velocities explored here, the resulting effects of physical processes that could have affected the global properties of these galaxies (outflows, inflows) are independent of halo mass. Our sample also supports galaxy-togalaxy scatter in the condensed baryon fraction up to a factor of two. Untangling the observational and intrinsic scatter is difficult with the current data, but manifestly a next avenue that needs to be explored.

Our value of the BTF slope agrees with certain previous studies (Avila-Reese et al. 2008; Hall et al. 2012) and we present one possible explanation for the disagreement with other studies that found steeper slopes (McGaugh 2012). We suggest that the disagreement comes about from the normalization of the stellar mass estimation. While we do not have direct evidence favoring one mass normalization over another, we prefer ours because it results in closer agreement in the derived BTF relations when using independent samples of gas- and star-dominated galaxies. We show that combining the gas- and star-dominated galaxy samples, when different stellar mass normalizations are used, can result in slope differences consistent with what is found among the various studies. Our data also result in excellent agreement with the recent simulations of Aumer et al. (2013).
Although that agreement cannot be used to support our stellar mass estimates, it does suggest that simulations are approaching a level of sophistication rivaling the observational uncertainties in determining the internal structural properties of individual galaxies. Finally, and perhaps eventually most illuminating when the origin is understood, we note the continuing tension between the BTF results, which suggests a constant $M_{\text {baryon }} / M_{\text {halo }}$, and those from statistical studies of galaxies, such as abundance matching models, that find a decreasing $M_{\text {baryon }} / M_{\text {halo }}$ with decreasing $M_{\text {halo }}$.

D.Z. acknowledges financial support from NASA ADAP NNX12AE27G and NSF AST-1311326, and thanks NYU CCPP for its hospitality during long-term visits. The authors thank Michael Aumer for sending us the baryonic masses and circular velocities of simulated galaxies that we incorporated into Figure 8, and Marc Verheijen and R. Brent Tully for comments on the manuscript. We also gratefully acknowledge discussions with Stacy McGaugh, which led both to improvements of the current manuscript and to related ideas to pursue in subsequent work. H.C. and J.S. acknowledge support from the Lyon Institute of Origins under grant ANR-10-LABX-66. The authors acknowledge the support from the FP7 Marie Curie Actions of the European Commission, via the Initial Training Network DAGAL under REA grant agreement PITN-GA-2011-289313. The authors thank the entire $S^{4} \mathrm{G}$ team for the efforts in making this program possible. K.S., J-C.M-M, and T.K acknowledge support from the National Radio Astronomy Observatory is a facility of the National Science Foundation operated under cooperative agreement by Associated Universities, Inc. This research has made use of the NASA/IPAC Extragalactic Database (NED), which is operated by the Jet Propulsion Laboratory, California Institute of Technology, under contract with NASA. This research is based in part on observations made with the Spitzer Space Telescope, which is operated by the Jet Propulsion Laboratory, California Institute of Technology under a contract with NASA.

\section{REFERENCES}

Aumer, M., White, S. D. M., Naab, T., \& Scannapieco, C. 2013, MNRAS, 434,3142

Avila-Reese, V., Zavala, I., Firmani, C., \& Hernández-Toledo, H. M. 2008, AJ, 136,1340

Barber, C., Starkenburg, E., Navarro, J., McConnachie, A., \& Fattahi, A. 2014, MNRAS, 437, 959

Bell, E. F., \& de Jong, R. S. 2001, ApJ, 550, 212

Benson, A. J., Cole, S., Frenk, C. S., Baugh, C. M., \& Lacey, C. G. 2000, MNRAS, 311, 793

Berlind, A., \& Weinberg, D. H. 2002, ApJ, 575, 587

Boylan-Kolchin, M., Bullock, J. S., Sohn, S. T., Besla, G., \& van der Marel, R. P. 2013, ApJ, 768, 140

Bullock, J. S., Dekel, A., Kolatt, T. S., et al. 2001, ApJ, 555, 240

Burstein, D., Bender, R., Faber, S., \& Nolthenius, R. 1997, AJ, 114, 1365

Buta, R., Sheth, K., Regan, M., et al. 2010, ApJS, 190, 147

Cappellari, M., McDermid, R. M., Alatalo, K., et al. 2012, Natur, 484, 485

Carignan, C., \& Beaulieu, S. 1998, ApJ, 347, 760

Comerón, S., Elmegreen, B. G., Salo, H., et al. 2012, ApJ, 759, 98

Conroy, C., Gunn, J. E., \& White, M. 2009, ApJ, 299, 486

Courteau, S. 1997, AJ, 114, 2402

Courteau, S., \& Rix, H. W. 1999, ApJ, 513, 561

Courtois, H. M., Tully, R. B., FIsher, J. R., et al. 2009, AJ, 138, 1938

Courtois, H. M., Tully, R. B., Makarov, D. I., et al. 2011, MNRAS, 414, 2005

Cybulski, R., Yun, M. S., Fazio, G. G., \& Gutermuth, R. A. 2014, MNRAS, in press

de Vaucouleurs, G., de Vaucouleurs, A., Corwin, H. G., et al. 1991, Third Reference Catalogue of Bright Galaxies (RC3) (New York: Springer)

Djorgovski, S., \& Davis, M. 1987, ApJ, 313, 59 
Dressler, A., Lynden-Bell, D., Burstein, D., et al. 1987, ApJ, 313, 42

Eskew, M., Zaritsky, D., \& Meidt, S. 2012, AJ, 143, 139

Fazio, G. G., Hora, J. L., Allen, L. E., et al. 2004, ApJS, 154, 10

Freedman, W. L., \& Madore, B. F. 2010, ARA\&A, 48, 673

Freedman, W. L., Madore, B. F., Gibson, B. K., et al. 2001, ApJ, 553, 47

Freeman, K. C. 1999, in ASP Conf. Ser. 170, The Low Surface Brightness Universe, ed. J. I. Davies, C. Impey, \& S. Phillips (San Francisco, CA: ASP), 3

Gao, L., \& White, S. D. M. 2007, MNRAS, 377L, 5

Geha, M., Blanton, M. R., Masjedi, M., \& West, A. A. 2006, ApJ, 653, 240

Gil de Paz, A., Madore, B. F., Boissier, S., et al. 2005, ApJL, 627, L29

Gnedin, O. Y., Weinberg, D. H., Pizagno, J., Prada, F., \& Rix, H.-W. 2007, ApJ, 671,1115

Governato, F., Willman, B., Mayer, L., et al. 2007, MNRAS, 374, 1479

Guo, Q., White, S., Li, C., \& Boylan-Kolchin, M. 2010, MNRAS, 404, 1111

Gurovich, S., Freeman, K., Jerjen, H., Staveley-Smith, L., \& Puerari, I. 2010, AJ, 140,663

Hall, M., Courteau, S., Dutton, A. A., McDonald, M., \& Yucong, Z. 2012, MNRAS, 425, 2741

Harris, J., \& Zaritsky, D. 2009, AJ, 138, 1243

Herbert-Fort, S., Zaritsky, D., Moustakas, J., et al. 2012, ApJ, 754, 110

Hinshaw, G., Larson, D., Komatsu, E., et al. 2013, ApJS, 208, 19

Ho, L. C. 2007, ApJ, 669, 821

Holwerda, B., Muňoz-Mateos, J.-C., Comerón, S., et al. 2014, ApJ, 781, 12

Isobe, T., Feigelson, E. D., Akritas, M., \& Babu, G. J. 1990, ApJ, 364, 104

Kassin, S., Weiner, B. J., Faber, S. M., et al. 2007, ApJL, 660, L35

Kauffmann, G., Nusser, A., \& Steinmetz, M. 1997, MNRAS, 286, 795

Leroy, A. K., Walter, F., Brinks, E., et al. 2008, AJ, 136, 2782

Maraston, C., Daddi, E., Renzini, A., et al. 2006, ApJ, 652, 85

Mayer, L., \& Moore, B. 2004, MNRAS, 354, 477

McGaugh, S. S. 2005a, ApJ, 632, 859

McGaugh, S. S. 2005b, PhRvL, 95, 171302

McGaugh, S. S. 2012, AJ, 143, 40

McGaugh, S. S., \& de Blok, W. J. G. 1997, ApJ, 481, 689

McGaugh, S. S., \& de Blok, W. J. G. 1998, ApJ, 499, 41

McGaugh, S. S., Schombert, J. M., Bothun, G. D., \& de Blok, W. J. G. 2000, ApJL, 533, L99

Meidt, S. E., Schinnerer, E., Knapen, J. H., et al. 2012, ApJ, 744, 17

Meixner, M., Gordon, K. D., Indebetouw, R., et al. 2006, AJ, 132, 2268

Ménard, B., Scranton, R., Fukugita, M., \& Richards, G. 2010, MNRAS, 405, 1025

Meurer, G. R., Carignan, C., Beaulieu, S. F., \& Freeman, K. C. 1996, AJ, 111,1551

Mo, H., van den Bosch, F., \& White, S. D. M. 2010, Galaxy Formation and Evolution (Cambridge: Cambridge Univ. Press)

Nelson, A. E., Zaritsky, D., \& Cutri, R. M. 1998, AJ, 115, 2273

Papastergis, E., Cattaneo, A., Huang, S., Giovanelli, R., \& Haynes, M. P. 2012, ApJ, 759, 138
Persic, M., \& Salucci, P. 1991, MNRAS, 248, 325

Pfenniger, D., \& Revaz, Y. 2005, A\&A, 431, 511

Sheth, K., Regan, M., Hinz, J. L., et al. 2010, PASP, 122, 1397

Sorce, J. G., Courtois, H. M., Tully, R. B., et al. 2013, ApJ, 765, 94

Sprayberry, D., Bernstein, G. M., Impey, C. D., \& Bothun, G. D. 1995, ApJ, 438, 72

Stark, D. V., McGaugh, S. S., \& Swaters, R. A. 2009, AJ, 138, 392

Steinmetz, M., \& Navarro, J. F. 1999, ApJ, 513, 555

Strader, J., Caldwell, N., \& Seth, A. C. 2011, AJ, 142, 8

Strauss, M. A., \& Willick, J. A. 1995, PhR, 261, 527

Tal, T., \& van Dokkum, P. G. 2011, ApJ, 731, 89

Thilker, D. A., Bianchi, L., Boissier, S., et al. 2005, ApJL, 619, L79

Tinker, J. L., Conroy, C., et al. 2008, ApJ, 686, 53

Trachternach, C., de Blok, W. J. G., McGaugh, S. S., van der Hulst, J. M., \& Dettmar, R.-J. 2009, A\&A, 505, 577

Treu, T., Auger, M. W., Koopmans, L. V. E., et al. 2010, ApJ, 709, 1195

Tully, R. B., \& Courtois, H. M. 2012, ApJ, 749, 78

Tully, R. B., \& Fisher, J. R. 1977, A\&A, 54, 661

Tully, R. B., Rizzi, L., Shaya, E. J., et al. 2009, AJ, 138, 323

van Dokkum, P. G., \& Conroy, C. 2010, Natur, 468, 940

Verheijen, M. 2001, ApJ, 563, 694

Walker, M. 1999, MNRAS, 308, 551

Watkins, L. L., Wyn Evans, N., \& An, J. H. 2010, MNRAS, 406, 264

Weiner, B., Willmer, C. N. A., Faber, S. M., et al. 2006, ApJ, 653,1049

Werner, M. W., Roellig, T. L., Low, F. J., et al. 2004, ApJS, 154, 1

White, M., Hernquist, L., \& Springel, V. 2001, ApJL, 550, L129

Yang, X., Mo, H. J., Jing, Y. P., van den Bosch, F. C., \& Chu, Y. 2004, MNRAS, 350, 1153

Yegorova, I. A., \& Salucci, P. 2007, MNRAS, 377, 507

Young, J. S., \& Knezek, P. 1989, ApJL, 347, L55

Young, J. S., \& Scoville, N. Z. 1991, ARA\&A, 29, 581

Zaritsky, D. 1994, AJ, 108, 1619

Zaritsky, D. 2012, ISRAA, 2012, 12

Zaritsky, D., \& Christlein, D. 2007, AJ, 134, 135

Zaritsky, D., Colucci, J. E., Pessev, P. M., Bernstein, R. A., \& Chandar, R. 2012, ApJ, 761, 93

Zaritsky, D., Colucci, J. E., Pessev, P. M., Bernstein, R. A., \& Chandar, R. 2013, ApJ, 770, 121

Zaritsky, D., Gonzalez, A. H., \& Zabludoff, A. I. 2006a, ApJL, 642, L37

Zaritsky, D., Gonzalez, A. H., \& Zabludoff, A. I. 2006b, ApJ, 638, 725

Zaritsky, D., Olszewski, E. W., Schommer, R. A., Peterson, R. C., \& Aaronson, M. 1989, ApJ, 345, 759

Zaritsky, D., Salo, H., Laurikainen, E., et al. 2013, ApJ, 772, 135

Zaritsky, D., Zabludoff, A. I., \& Gonzalez, A. H. 2008, ApJ, 682, 68

Zhu, G., Zheng, Z., Lin, W. P., et al. 2006, ApJL, 639, L5

Zwaan, M. A., van der Hulst, J. M., de Blok, W. J. G., \& McGaugh, S. S. 1995, MNRAS, 273, L35 